\title{
Health impact of pain in the hip region with and without radiographic evidence of osteoarthritis: a study of new attenders to primary care
}

Fraser Birrell, Peter Croft, Cyrus Cooper, Gillian Hosie, Gary Macfarlane, Alan Silman, on behalf of the PCR Hip Study Group

\begin{abstract}
Objectives-To assess the health impact of hip pain at the time of first presentation to primary care, and the influence on this of radiographic evidence of osteoarthritis.

Subjects and methods-Cross sectional survey of 195 patients (63 male, 132 female), aged 40 years and over, presenting with a new episode of hip pain, recruited from 35 general practices across the UK. Health status at presentation was determined by a structured questionnaire on symptoms, healthcare use, and health related quality of life (SF-36). Pelvic radiographs were assessed blindly for hip osteoarthritis using standard scoring systems.
\end{abstract}

Results-The overall impact on health was substantial. Before their first consultation, three quarters of patients needed analgesics, half used topical creams or ointments, and one in eight used a walking stick. Most of these impact measures were, however, unrelated to the degree of radiographic change, though use of a walking stick was increased in those with the most severe damage. Health status, as judged by the SF-36, was also impaired for measures of physical function and pain, but the impact on the "mental health", "general health", and "vitality" dimensions was small. There was a weak relation between the SF-36 scores and radiographic change, with many domains unrelated to the severity of radiographic damage.

Conclusions-This study is the first to show the therapeutic impact and pattern of impairment in health status resulting from hip pain at the time of first presentation to the healthcare services. Unlike many regional pain syndromes seen in primary care, such as back pain, hip pain does not impact on wider aspects of quality of life, such as general health status, mental health, or vitality. Furthermore, any impact of hip pain in this group is not markedly influenced by the degree of structural damage. Further follow up is required to determine whether such damage influences the persistence of any adverse impact.

(Ann Rheum Dis 2000;59:857-863)

Although there have been studies investigating the health impact of hip osteoarthritis (OA) at the time of total hip arthroplasty, ${ }^{1}$ the decision to perform total hip arthroplasty in patients with OA of the hip is based largely on the patient's report of pain and disability, usually with demonstrable structural change. ${ }^{2-4}$ There has, however, been little research examining the impact of hip disease at the time of first presentation to primary care. This may be important for the following reasons. Health status of early disease can offer insight into the need for arthroplasty at this stage. Secondly, at the time of hospital referral, most patients have severe disease and the natural history before referral is unknown. ${ }^{5}$ Thirdly, the inherent selection bias of hospital based studies does not permit any analysis of the relative contributions of pain and structural (that is, radiographic) damage on impact.

The objective of this study was to answer two questions: firstly, what is the impact on health status of hip pain in patients newly presenting in primary care and, secondly, is impact increased in those with radiographic hip OA compared with those with other causes for their hip pain?

\section{Subjects and methods}

SUBJECTS

This was a multicentre study of patients presenting with new episodes of hip pain in primary care. In all, 36 general practitioner (GP) members of the Primary Care Rheumatology Society, a group of doctors who have a particular interest in musculoskeletal pain, were recruited from all over the United Kingdom. They were asked to enrol consecutive new attenders aged 40 years or older with hip pain. The practices were divided between urban and rural areas and covered a broad socioeconomic spectrum. Ethical permission was secured locally for each practice before the study started.

As no standardised definition for hip pain exists, a working case definition was derived by a consensus group including the study coordinators and GPs and validated by pilot testing. This was defined as pain within a preshaded area on a standardised pain drawing (fig 1) which, on GP clinical assessment, was not thought to originate from structures outside the hip (for example, low back, trochanteric bursa). Use of a pictorial instrument of the area to be regarded as "hip pain" enhanced the standardisation of case definition. Patients presenting with pain in a hip with prior arthroplasty were excluded.

The aim was to study early disease severe enough to prompt a visit to the doctor. As hip pain may be a recurrent problem throughout 
Back

Left

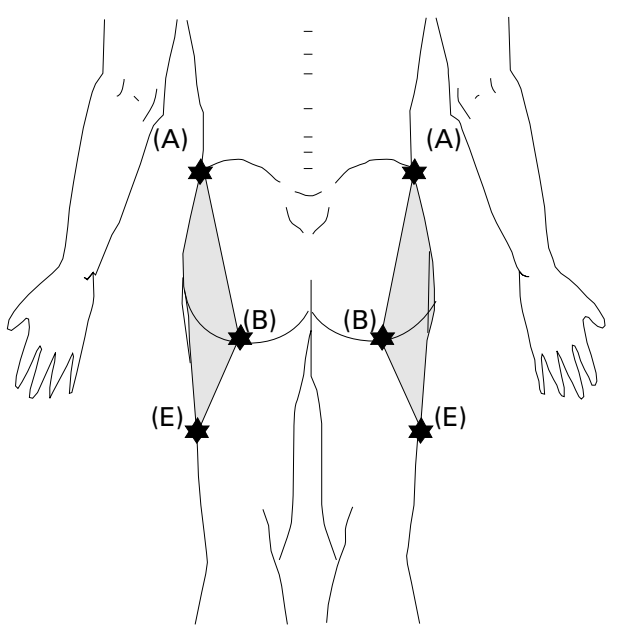

Front

Right
Left

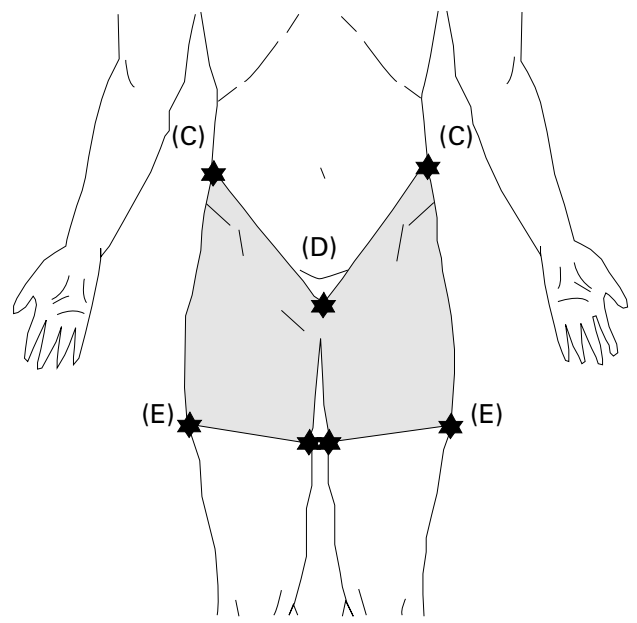

\begin{tabular}{|ll|}
\hline Landmarks: & A - lliac crest \\
& B - Ischial tuberosity \\
& C - Anterior superior iliac spine \\
& D - Pubic tubercle \\
& E - $1 / 3$ way down thigh \\
\hline
\end{tabular}

Figure 1 Standardised drawing for hip pain.

life, the definition of a new episode presented obvious difficulties and thus a pragmatic approach was adopted. Patients were excluded if they either had consulted at any time in the past for their current pain; or had consulted with any hip pain in the previous 12 months, even if not related to their current pain. A subset of the medical records of the patients recruited was reviewed to validate the case entry criteria and to provide an estimate of the proportion of the study group who were first-ever consulters.

\section{METHODS}

The patient completed a structured questionnaire at presentation. Pain severity was measured using a numerical rating scale (1-10). This approach is well validated, performing comparably or better than other pain rating instruments. ${ }^{6}$ Duration of hip pain was measured by recalled interval since the current episode started. There were closed questions on current physical activities, including walking, and treatments or assistance sought for the hip pain leading to presentation. Health related quality of life was assessed using the Short Form 36 Health Questionnaire (SF-36), ${ }^{7}$ to which responses were coded and transformed according to standard algorithms. ${ }^{8}$

An anteroposterior pelvis radiograph was requested on each subject, with a set of standard instructions given to the local $x$ ray department. As there is no consensus definition for radiographic $\mathrm{OA},{ }^{9} x$ rays were graded for both Croft's modification of the Kellgren and Law- rence grade ("Croft grade") and minimum joint space. ${ }^{10}$ Gradings were made by two independent observers, unaware of the clinical status of the subject, with adjudication of any discrepancies made by a third observer.

\section{ANALYSIS}

The impact of hip pain was expressed in terms of pain severity, walking, treatments, and health professionals seen, stratified by age group and sex. The influence of radiographic hip OA was assessed after stratification into three groups for each of the Croft grades $(0-1$, $2-3,4-5)$ and for minimum joint space $(>2.5$ $\mathrm{mm}, 1.5-2.5 \mathrm{~mm}, \leqslant 1.5 \mathrm{~mm})$. The association between radiographic grade and specific health outcomes was calculated by logistic regression, taking the least severe group (Croft grade $<2$, minimum joint space $>2.5 \mathrm{~mm}$ ) as the referent group. All analyses were adjusted for age and sex.

The crude scores for the individual domains are difficult to interpret. Thus the health related quality of life data for the whole group were compared with normative population data for each of the individual domains of the SF-36. In the absence of published reference ranges for the normal population by age and sex in the UK, we used data gathered from a population survey of over 3000 subjects (response rate $88 \%$ ) from a primary care register, conducted by the authors for another study. These data were then used to derive sex and decade-of-age specific estimates of the mean and standard deviation of SF-36 scores 
Table 1 Major clinical characteristics of cohort. Results are shown as No (\%)

\begin{tabular}{lc}
\hline Characteristic & Frequency \\
\hline Side of pain: & $103(53)$ \\
$\quad$ Right & $87(45)$ \\
Left & $5(3)$ \\
Both & \\
Pain duration* (months) & $58(30)$ \\
$\quad 33$ & $80(41)$ \\
$3-12$ & $54(28)$ \\
$>12$ & $74(38)$ \\
& \\
Reported previous episodes of hip pain & \\
& \\
Radiographic grade-modified Kellgren and Lawrence & $83(43)$ \\
0 & $26(13)$ \\
1 & $19(10)$ \\
2 & $34(17)$ \\
4 & $22(11)$ \\
5 & $11(6)$ \\
Radiographic grade-minimum joint space (mm) & $136(70)$ \\
$>2.5$ & $31(16)$ \\
$>1.5, \leqslant 2.5$ & $28(14)$ \\
\hline 1.5 &
\end{tabular}

*Pain duration was not available for three patients, hence total not $100 \%$.

Table 2 Health status scores-SF-36

\begin{tabular}{lc}
\hline$S F-36$ measure & Median score $\left(I Q R^{\star}\right)$ \\
\hline Physical functioning & $55(35-75)$ \\
Physical role & $25(0-75)$ \\
Bodily pain & $45(32-55)$ \\
General health & $65(50-77)$ \\
Vitality & $55(40-70)$ \\
Social function & $75(50-100) \dagger$ \\
Emotional role & $100(33-100) \dagger$ \\
Mental health & $76(60-88)$ \\
\hline
\end{tabular}

${ }^{\star} \mathrm{IQR}=$ interquartile range.

†The $75^{\text {th }}$ centile coincided with the best possible score.

for each domain. The observed SF-36 domain scores for the patients with hip pain were then $\mathrm{Z}$ transformed. Thus the scores for each subject were calculated as the number of standard deviations away from the age decade and sex specific mean in the normal population. The advantage of this $\mathrm{Z}$ standardised score, apart from allowing for age and sex, is that it permits a direct comparison of the results between the eight individual domains. These $\mathrm{Z}$ scores were then compared between the radiographic groups within the study
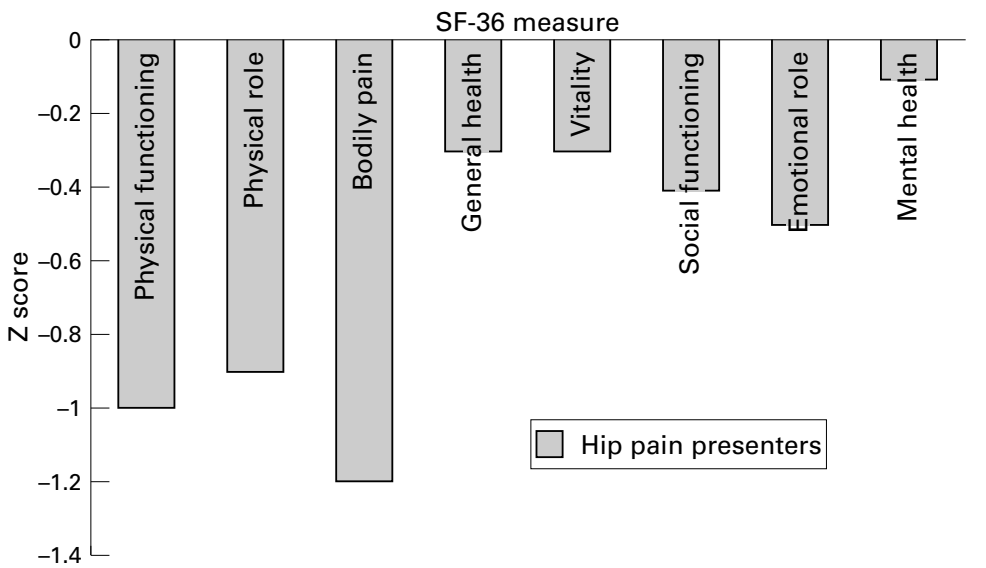

Figure 2 SF-36 measures: depression of $Z$ score compared with age and sex matched norms. group. Analysis was performed with Stata statistical software. ${ }^{11}$

\section{Results}

The study recruited 195 patients, of whom 132 $(68 \%)$ were female, with a mean (SD) age of 63 (11) years. Table 1 summarises the major clinical characteristics of the cohort. The majority had unilateral pain, with a small right sided predominance.

Any patient who had previously attended during the current episode had been excluded from our study group. There was, however, a broad range of duration of pain before this consultation. Thirty per cent of the patients had pain duration of less than three months after the onset at the time of first attendance. The study was designed to be an investigation of new episodes of hip pain and thus patients were not eligible for inclusion if they recalled any hip pain in the previous 12 months, even if such an episode was distinct from the current pain of the patients recruited. However, 74 $(38 \%)$ recalled a previous episode, distinct from the current pain at least one year previously. A review of the subsample of their GP records suggested that only a quarter of that $38 \%$ had ever consulted with hip pain. These results thus imply that approximately $90 \%$ of our study cohort were consulting for the first time with any hip pain.

The health impact was first examined by the SF-36. Table 2 shows the SF-36 scores by domain, and fig 2 a comparison with normative data, expressed as a reduction of the $Z$ score from age and sex matched normative data. The $\mathrm{Z}$ score was depressed across most domains, indicating health impairment in these subjects presenting with hip pain. The most striking impact was seen for physical measures"physical functioning" and "physical role" as well as "pain". However, the impact on "mental health", "general health", and "vitality" was small. The impact of hip pain was also analysed in relation to specific measures obtained for the hip. Table 3 shows these results for pain severity, current walking, self treatment, and health professionals seen, stratified by age and sex. The overall impact was substantial-one in eight had used a walking stick, three quarters had taken analgesics, and half had used topical creams or ointments, before their first attendance. The impact was surprisingly not greater in those above median age. The only exception was that the older age group were more likely to use a walking stick.

Almost half the patients had definite evidence of radiographic change (Croft grade $>1$ ) and a substantial minority had advanced $x$ ray changes (table 1). Figure 3 shows the relation between SF-36 Z scores and radiographic severity stratified by Croft grade (fig $3 \mathrm{~A}$ ) and minimum joint space (fig 3B). There was no overall consistent pattern of increasing impact with increasing radiographic severity. The pain scale scores were not related to the degree of radiographic damage. By contrast, the functional scale scores did show the greatest 
impairment in those with the most damage. No other domains showed this pattern.

Tables 4 and 5 show the relation between the other impact measures and radiographic change by Croft grade and minimum joint space, respectively. These data show, as with the SF-36, that independent of grading scheme, the impact measures studied were broadly similar across the grades of radiographic damage. However, both minimum joint space of $1.5 \mathrm{~mm}$ or less and Croft grades of 4-5 are associated with a threefold increased odds of using a walking stick, after adjustment for age and sex. There was also a doubling of the odds of pain severity score above the median, though this was not statistically significant.

\section{Discussion}

We have shown that there is a substantial impact from hip pain, even at first presentation to primary care with this problem. The most striking impact on health related quality of life was seen for physical measures of the SF-36physical functioning, physical role, and pain, but the impact on mental health, general health, and vitality, when compared with normative data, was small. Further, one in
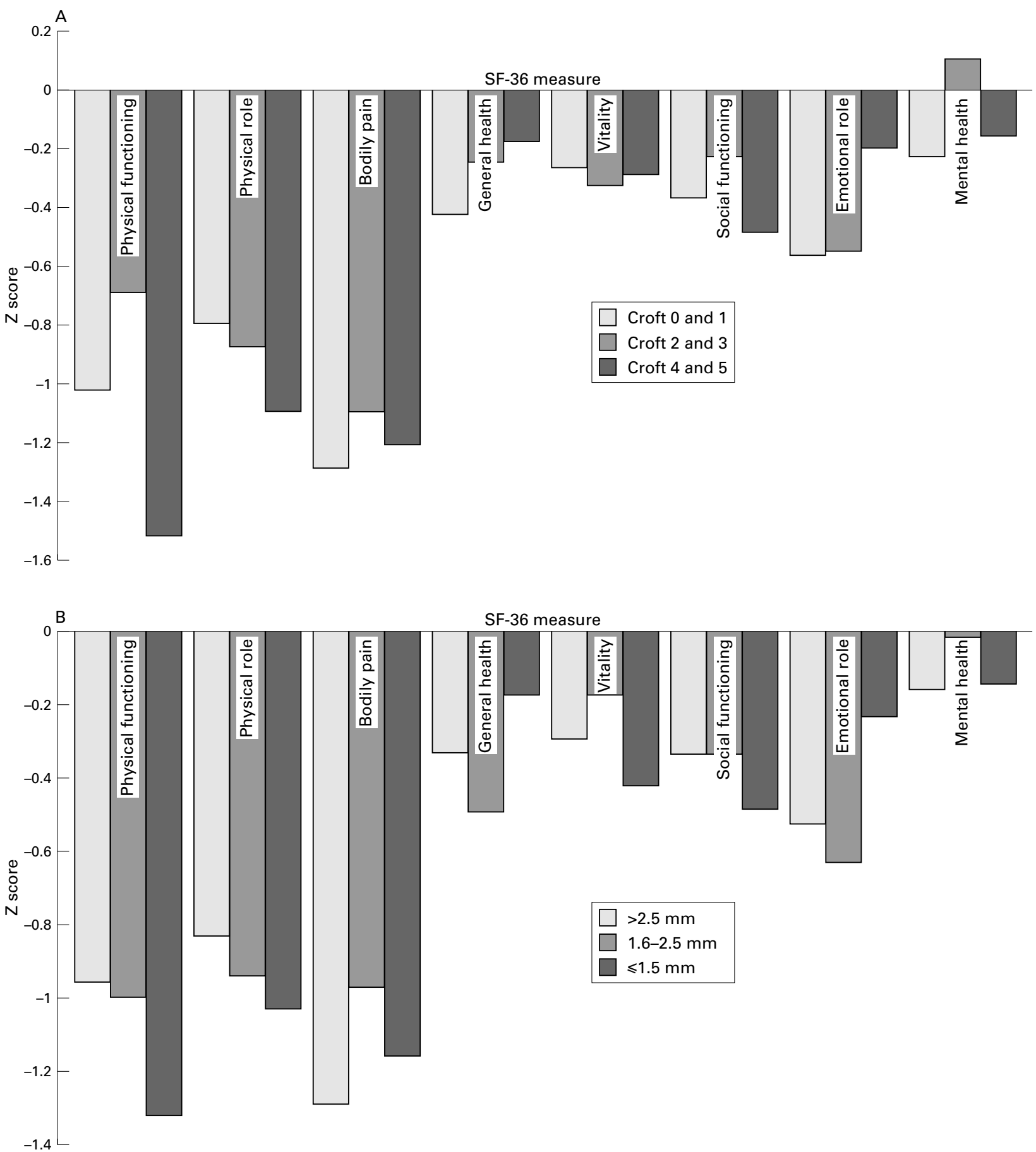

Figure 3 SF-36 measures by radiographic grade stratified by (A) Croft grade and (B) by minimum joint space—depression of $Z$ score. 
Table 3 Impact of hip pain at first attendance. Results shown as No (\%) except for pain severityt

\begin{tabular}{|c|c|c|c|c|c|}
\hline & \multicolumn{5}{|c|}{ Impact of hip pain by age and sex } \\
\hline & \multirow[b]{2}{*}{ All $(n=195)$} & \multicolumn{2}{|l|}{ Age group } & \multicolumn{2}{|l|}{$\operatorname{Sex}$} \\
\hline & & $40-63(n=98)$ & $64+(n=97)$ & Male $(n=63)$ & Female $(n=132)$ \\
\hline Median (IQR) pain severity & $5(3-7)$ & $5(3-7)$ & $5(3-7)$ & $4(3-7)$ & $5(3-7)$ \\
\hline Walk $>30 \mathrm{~min} /$ day & $130(68)$ & $71(74)$ & $59(61)$ & $49(78)$ & $81(63)$ \\
\hline Used a walking stick & $23(12)$ & $6(6)$ & $17(18)$ & $13(21)$ & $10(8)$ \\
\hline Taken analgesics & $141(73)$ & $72(75)$ & $69(71)$ & $38(60)$ & $103(79)$ \\
\hline Used topical treatment & $94(46)$ & $44(46)$ & $50(52)$ & $38(60)$ & $56(43)$ \\
\hline Consulted physiotherapist & $15(8)$ & $6(6)$ & $9(9)$ & $8(13)$ & $7(5)$ \\
\hline Consulted osteopath/chiropractor & $15(8)$ & $10(10)$ & $5(5)$ & $3(5)$ & $12(9)$ \\
\hline
\end{tabular}

$\star \mathrm{IQR}=$ interquartile range.

† Percentages reflect subjects with full data for each category

Table 4 Logistic regression of the impact of symptoms by grade of osteoarthritis $\neq$

\begin{tabular}{|c|c|c|c|c|c|c|c|}
\hline & \multicolumn{6}{|c|}{${ }^{\star}$ Croft grade group } & \multirow[b]{3}{*}{$\chi^{2}$ trend $p<$} \\
\hline & \multicolumn{2}{|c|}{$0-1(n=109)$} & \multicolumn{2}{|c|}{$2-3(n=53)$} & \multicolumn{2}{|c|}{$4-5(n=33)$} & \\
\hline & No (\%) & $\begin{array}{l}\text { ORt } \\
\text { Reference }\end{array}$ & No (\%) & ORt $(95 \% C I)$ & No (\%) & $O R+(95 \% C I)$ & \\
\hline Pain severity $>4$ & $52(49)$ & 1 & $28(56)$ & $1.3(0.6$ to 2.5$)$ & $20(63)$ & $1.8(0.8$ to 4.1$)$ & 0.19 \\
\hline Walk >30 $\mathrm{min} /$ day & $79(74)$ & 1 & $30(58)$ & $0.5(0.3$ to 1.1$)$ & $21(64)$ & $0.6(0.3$ to 1.4$)$ & 0.14 \\
\hline Use a walking stick & $9(8)$ & 1 & $5(9)$ & $1.0(0.3$ to 3.2$)$ & $9(27)$ & $3.3(1.1$ to 9.4$)$ & 0.01 \\
\hline Take analgesics & $77(72)$ & 1 & $36(68)$ & $0.8(0.4$ to 1.7$)$ & $28(85)$ & $2.7(0.9$ to 7.9$)$ & 0.21 \\
\hline Use topical treatments & $50(47)$ & 1 & $25(47)$ & $1.0(0.5$ to 2.0$)$ & $19(58)$ & $1.4(0.6$ to 3.1$)$ & 0.29 \\
\hline See physiotherapist & $8(7)$ & 1 & $3(6)$ & $0.7(0.2$ to 2.9$)$ & $4(12)$ & $1.4(0.4$ to 5.3$)$ & 0.51 \\
\hline See osteopath/chiropractor & $8(7)$ & 1 & $5(9)$ & $1.3(0.4$ to 4.4$)$ & $2(6)$ & $0.8(0.2$ to 4.0$)$ & 0.96 \\
\hline
\end{tabular}

${ }^{\star}$ Croft's modification of the Kellgren and Lawrence grade.

†Adjusted for age and sex.

$\ddagger$ Percentages reflect subjects with full data for each category.

Table 5 Logistic regression of the impact of symptoms by minimal joint spacet

\begin{tabular}{|c|c|c|c|c|c|c|c|}
\hline & \multicolumn{6}{|c|}{ Minimal joint space group (mm) } & \multirow[b]{3}{*}{$\chi^{2}$ trend $p<$} \\
\hline & \multicolumn{2}{|c|}{$>2.5(n=136)$} & \multicolumn{2}{|c|}{$>1.5, \leqslant 2.5(n=31)$} & \multicolumn{2}{|c|}{$\leqslant 1.5(n=28)$} & \\
\hline & No $(\%)$ & $\begin{array}{l}\text { OR } \\
\text { reference }\end{array}$ & No $(\%)$ & $O R^{\star}(95 \% C I)$ & No $(\%)$ & $O R^{\star}(95 \% C I)$ & \\
\hline Pain severity $>4$ & $65(48)$ & 1 & $17(55)$ & $1.1(0.5$ to 2.5$)$ & $18(64)$ & $2.0(0.8$ to 4.8$)$ & 0.10 \\
\hline Walk >30 min/day & $96(72)$ & 1 & $17(55)$ & $0.6(0.3$ to 1.3$)$ & $17(61)$ & $0.7(0.3$ to 1.6$)$ & 0.14 \\
\hline Use a walking stick & $13(10)$ & 1 & $2(6)$ & $0.6(0.1$ to 3.0$)$ & $8(29)$ & $3.0(1.0$ to 8.6$)$ & 0.02 \\
\hline Take analgesics & $98(73)$ & 1 & $21(68)$ & $0.7(0.3$ to 1.7$)$ & $22(79)$ & $1.5(0.5$ to 4.2$)$ & 0.65 \\
\hline Use topical treatments & $62(46)$ & 1 & $16(52)$ & $1.3(0.6$ to 2.9$)$ & $16(57)$ & $1.5(0.6$ to 3.4$)$ & 0.24 \\
\hline See physiotherapist & $11(8)$ & 1 & $1(3)$ & $0.4(0.0$ to 3.2$)$ & $3(11)$ & $1.1(0.3$ to 4.6$)$ & 0.91 \\
\hline See osteopath/chiropractor & $12(9)$ & 1 & $1(3)$ & $0.3(0.0$ to 2.8$)$ & $2(7)$ & $1.0(0.2$ to 4.9$)$ & 0.53 \\
\hline
\end{tabular}

^Adjusted for age and sex.

$\dagger$ Percentages reflect subjects with full data for each category.

eight had used a walking stick, three quarters had needed to take analgesics, and half had used topical creams or ointments. A small proportion had already seen a physiotherapist or osteopath, though referral by the GP was the usual sequence of events. The second objective of the study was to examine the influence of the occurrence and degree of radiographic damage on health impact. Surprisingly, perhaps, for most of the impact measures assessed there was little influence of radiographic grade. The patients were all new attenders to primary care with hip pain and, possibly, the threshold for attendance, either because of the physical distress or functional impairment, is similar and independent of the origin of the pain. These results describe the impact at presentation, however, and the role of radiographic status in predicting sustained impact on health will be ascertained by follow up of this cohort.

Certain methodological issues must be considered to put the study results in context.
Firstly, diagnosis of the hip as the anatomical source of pain is difficult owing to its site and deep location. Eligibility was based not only on the geographical location of the pain but also on the GP's clinical judgment that the pain arose from the hip joint itself. Thus we aimed at excluding pain that might be referred-for example, from the spine, or had arisen from structures adjacent to the hip joint-for example, trochanteric bursitis. The GP participants, all members of the Primary Care Rheumatology Society, were all experienced in hip joint assessment but in this study it is impossible to verify whether the pain actually arose from the hip joint itself. It is thus of interest that over $40 \%$ had radiographically normal hips. There are many non-osteoarthritic causes of hip pain, but there is clearly a potential for GP error in the assignment of the hip as the source of the pain.

Diagnosis in this multicentre study might have varied between GPs and over time, 
leading to differences in the threshold for recruitment. These differences were minimised by the series of consensus meetings before recruitment started. This definition used a pain drawing plus GP "clinical" diagnosis of pain coming from the hip joint itself to maximise comparability across several centres and over time. Isolated pain referred to the knee might have been missed by this definition, but this is rare and conclusions about this subgroup could only be drawn from a much larger study.

Secondly, the definition of a hip pain episode was somewhat arbitrary. As the focus was on new episodes provoking attendance, prior attendance with this episode of hip pain or recurrent attendance for hip pain within the previous 12 months were exclusion criteria. As shown most patients were indeed presenting for the first time.

Thirdly, the normative population data for the SF-36 scores were not derived from the same general practice population as the patients with hip pain. This source of normative data might have affected the $\mathrm{Z}$ score comparisons owing to socioeconomic or regional variation. This source of error was minimised by surveying two practices chosen to reflect a broad socioeconomic mix to estimate population values. The internal comparison by radiographic change would not have been affected, where standardisation to any population would eliminate the effects of age and sex.

Finally, errors may be introduced in radiographic grading, though these were minimised by using accepted techniques ${ }^{10}$ and arbitration of discrepant readings. Such random errors would be expected to reduce the power of a study to detect a true difference and hence should not affect positive study conclusions.

A number of other studies have aimed at investigating the impact of hip pain both in the general population ${ }^{12-15}$ and on case series of clinic attenders. ${ }^{16}{ }^{17}$ Such studies have not, however, incorporated independent pain and disability measures and their relation to radiographic change. They are therefore not directly comparable with our study of primary care attenders.

The SF-36 is a well recognised tool for studying health status used quite extensively in hospital based interventional studies on hip OA. Improvements after arthroplasty in various SF-36 domains, particularly pain, function, and overall health, have been seen, ${ }^{18}{ }^{19}$ but it is unknown whether these scores return to population normal levels. Expressing the individual domains as $\mathrm{Z}$ scores has two main advantages over conventional summary scoring. Firstly, this method allows for the variation in SF-36 measures with age and sex without multivariate adjustment. Secondly, it permits expression of scores parametrically, though the distribution of actual SF-36 measures is often not Gaussian.

Comparison with the general population using the SF-36 showed evidence of impairment in hip pain presenters across a wide variety of domains designed to sample aspects of health related quality of life. The greatest impairment in SF-36 scores in this study com- pared with population values was seen for physical functioning, physical role, and pain scores. It is interesting to compare published data from low back pain presenters in primary care with the hip pain presenters in the current study. Subjects with low back pain do have impaired scores on mental health, vitality, and general health measures, as well as more global depression of SF-36 scores. $^{20}$ The lack of impairment in mental and general health and vitality measures suggests that the effect of hip pain on physical function and role may be mediated differently from that of low back pain. The associations between psychological factors and hip pain in primary care are therefore likely to vary from those seen in other regional pain disorders. Thus there is an observed association between psychological distress and both the development ${ }^{21}$ and persistence $^{22}$ of low back pain, a relation that has yet to be shown for hip pain.

The relation of SF-36 domains to radiographic change was weak. Indeed, not all domains of the SF-36 were related to radiographic change. Mental health, general health, and vitality scores were little influenced. This confirms that different modalities are being measured by the SF-36, giving a broader view than the use of disease-specific disability measures alone.

\section{Conclusions}

This study is the first to show the impact and pattern of impairment in health status resulting from newly presenting hip pain. Unlike low back pain seen in primary care, hip pain associated with radiographic OA does not impact on general health status, mental health, or vitality. Furthermore, impairment of health related quality of life is only weakly associated with increasing radiographic change.

The cooperation of the patients and recruiting GPs from the Primary Care Rheumatology Society is much appreciated. We are also grateful to Liz Nahit, Linda Robinson, and Cara Afzal, for their unstinting efforts in recruitment and follow up of the for their unstintir
study patients.

The PCR Hip Study Group comprises Dr Martin Allen, Dr Trevor Birnie, Dr Fraser Birrell, Dr Susan Bradbury, Dr David Child, Dr A S Chopra, Professor Cyrus Cooper, Professor Peter Croft, Dr Tim Davies, Dr Beryl Dennis, Dr Elizabeth Dickson, Dr John Dickson, Dr Ian Louis Foster, Dr Roger Gadsby, Dr Iain Gilchrist, Dr Charmian Goldwyn, Dr Martin W Hall, Dr Iain Gilchrist, Dr Charmian Goldwyn, Dr Martin W Hall, Dr
Allan Harris, Dr B M Heap, Dr Stan G Hill, Dr M J L Hopkins, Allan Harris, Dr B M Heap, Dr Stan G Hill, Dr M J L Hopkins,
Dr Gill Hosie, Dr David Jobling, Dr Martin John, Dr Fred Dr Gill Hosie, Dr David Jobling, Dr Martin John, Dr Fred
Kavalier, Dr P Lindsay,Professor Gary Macfarlane, Dr John Kavalier, Dr P Lindsay,Professor Gary Macfarlane, Dr John
Machen, Dr Ian Robertson, Dr James D A Robertson, Dr Machen, Dr Ian Robertson, Dr James D A Robertson, Dr
Werner Rumfeld, Dr R W Shaw, Professor Alan Silman, Dr Ros Simpson, Dr Lorna K R Smith, Dr T G Stammers, Dr Michael Stevenson, Dr Anil Vaghmaria, Dr Hywel Watkin, Dr Andrew Watson, Dr David Wheeler, Dr Jane Wilcock, and Dr Michaela Young.

Funding: Arthritis Research Campaign, Chesterfield, United Kingdom. FNB is an ARC clinical epidemiology training fellow. Conflict of interest: None.

1 Stucki G, Liang MH, Phillips C, Katz JN. The Short Form-36 is preferable to the SIP as a generic health measure in patients undergoing elective total hip arthroplasty. Arthritis Care Research 1995;8:174-81.

2 Fox KM, Hochberg MC, Resnik CS, Kenzora JE, Hebel JR, Zimmerman SI, et al. Severity of radiographic findings in hip osteoarthritis associated with total hip arthroplasty. J hip osteoarthritis associated

$3 \mathrm{NIH}$ consensus development panel on total hip replacement. Total hip replacement. JAMA 1995;273:1950-6. 
4 Hadorn DC, Holmes AC. The New Zealand priority criteria project. Part 1: Overview. BMJ 1997;314:131-4.

5 Cushnaghan J, Dieppe P. Study of 500 patients with limb joint osteoarthritis. I. Analysis by age, sex, and distribution of symptomatic joint sites. Ann Rheum Dis 1991;50:8-13.

6 Jensen MP, Karoly P, Sanford B. The measurement of clinical pain intensity: a comparison of six methods. Pain 1986; 27:117-26.

7 Ware JB, Brook RH, Williams KN, Stewart AL, DaviesAvery A. Conceptualisation and measurement of health for adults in the health insurance study. Vol 1. Model of health and methodology. Santa Monica, California: Rand Corporation, 1980.

8 Ware JE. How to score the revised MOS Short-Form Health Scales. Boston, MA: Institute for the Improvement of Medical Care and Health, New England Medical Center, 1988.

9 Spector TD, Hochberg MC. Methodological problems in the epidemiological study of osteoarthritis. Ann Rheum Dis $1994 ; 53: 143-6$.

10 Croft P, Cooper C, Wickham C, Coggon D. Defining osteoarthritis of the hip for epidemiologic studies. Am J arthritis of the hip for ep

11 StataCorp. Stata statistical software: release 5.0 for Windows 95. College Station, TX: Stata Corporation, 1997

12 Wilcock GK. The prevalence of osteoarthrosis of the hip requiring total hip replacement in the elderly. Int J Epidemiol 1979;8:247-50

13 Fear J, Hillman M, Chamberlain MA, Tennant A Prevalence of hip problems in the population aged 55 years and over: access to specialist care and future demand for hip arthroplasty. Br J Rheumatol 1997;36:74-6.
14 Jørring K. Osteoarthritis of the hip. Acta Orthop Scand 1980;51:523-30.

15 Hubert HB, Bloch DA, Fries JF. Risk factors for physical disability in an aging cohort: the NHANES I Epidemiologic Followup Study. J Rheumatol 1993;20:480-8.

16 Danielsson LG. Incidence and prognosis of coxarthrosis. Acta Orthop Scand Suppl 1964;66:1-114.

17 Shepherd MM. Assessment of function after arthroplasty of the hip. J Bone Joint Surg Br 1954;36:354.

18 Kiebzak GM, Vain PA, Gregory AM, Mokris JG, Mauerhan DR. SF-36 general health status survey to determine patient satisfaction at short-term follow-up after total hip patient satisfaction at short-term follow-up after total hip and 72 .

19 Williams JI, Llewellyn Thomas H, Arshinoff R, Young N, Naylor CD. The burden of waiting for hip and knee replacements in Ontario. Ontario Hip and Knee Replacement Project Team. Journal of Evaluation in Cinical Practice 1997;3:59-68.

20 Garratt AM, Ruta DA, Abdalla MI, Buckingham JK, Russell IT. The SF36 Health Survey Questionnaire: an outcome measure suitable for routine use in the NHS? BMJ 1993;306:1440-4

21 Croft PR, Papageorgiou AC, Ferry S, Thomas E, Jayson MI, Silman AJ. Psychologic distress and low back pain. Evidence from a prospective study in the general population. Spine 1995;20:2731-7.

22 Thomas E, Silman AJ, Croft PR, Papageorgiou AC, Jayson MI, Macfarlane GJ. Predicting who develops chronic low back pain in primary care: a prospective study. BMJ 1999; 318:1662-7. 